\title{
The 3M Concept: Biomedical Translational Imaging from Molecules to Mouse to Man
}

\author{
Domokos Máthé ${ }^{1,2,3^{*}}$, Bálint Kiss², Bernadett Pályi ${ }^{4}$, Zoltán Kis ${ }^{4}$, László Forgách ${ }^{2}$, Nikolett Hegedüs², Zoltán \\ Varga $^{2}$, Krisztián Szigeti², Kinga Karlinger ${ }^{5}$, Miklós S Z Kellermayer ${ }^{2 *}$
}

\begin{abstract}
Imaging keeps pervading biomedical sciences from the nanoscale to the bedside. Connecting the hierarchical levels of biomedicine with relevant imaging approaches, however, remains a challenge.

Here we present a concept, called "3M", which can deliver a question, formulated at the bedside, across the wide-ranging hierarchical organization of the living organism, from the molecular level, through the small-animal scale, to whole-body human functional imaging. We present an example of nanoparticle development pipeline extending from atomic force microscopy to pre-clinical whole body imaging methods to highlight the essential features of the $3 \mathrm{M}$ concept, which integrates multi-scale resolution and quantification into a single logical process.

Using the nanoscale to human clinical whole body approach, we present the successful development, characterisation and application of Prussian Blue nanoparticles for a variety of imaging modalities, extending it to isotope payload quantification and shape-biodistribution relationships.

The translation of an idea from the bedside to the molecular level and back requires a set of novel combinatorial imaging methodologies interconnected into a logical pipeline. The proposed integrative molecules-to-mouse-to-man (3M) approach offers a promising, clinically oriented toolkit that lends the prospect of obtaining an ever-increasing amount of correlated information from as small a voxel of the human body as possible.
\end{abstract}

Keywords: nanoparticles, Prussian Blue, atomic force microscopy, SPECT/CT, biodistribution

${ }^{1}$ In Vivo Imaging Advanced Core Facility,

Semmelweis University Site, Hungarian Center of Excellence for Molecular Medicine (HCEMM),

Szeged, Hungary

${ }^{2}$ Department of Biophysics and Radiation

Biology, Semmelweis University, Budapest,

Hungary

${ }^{3}$ CROmed Translational Research Centres Ltd., Budapest, Hungary

${ }^{4}$ National Biosafety Laboratory, National Public Health Center, Budapest, Hungary

${ }^{5}$ Department of Clinical Imaging, Semmelweis

University, Budapest, Hungary

*Corresponding author: domokos.mathe@

cromedresearch.com

kellermayer.miklos@med.semmelweis-univ.hu

DOI: $10.2478 /$ ebtj-2021-0024

(C) 2021 Authors. This work was licensed under the Creative Commons Attribution-NonCommercialNoDerivs 4.0 License.

\section{Introduction}

"Seeing is believing", proclaims the age-old saying. Biomedicine is being pervaded by an ever-increasing number of imaging techniques with good reason. Imaging methods hold the prospect of providing spatial and temporal information and insights about physical-chemical fields such as concentration, mechanics and electric field. The ultimate aim is to be able to obtain and display as much spatio-temporally correlated information as possible about as small a volume element of the living organism as possible. In this short communication we provide an example of integrating macromolecular measurements and in vivo image-based quantification into a translational, therapeutic and diagnostic (hence theranostic) concept that holds important medical application prospects.

Modern biotechnology extensively applies nanomaterials of different structure and size as tools of delivery and effect enhancement (1). Translation from the bed to bench and back is the most important aim of all biotechnology developments. Usually, translation ends at the clinical prototype stage enabling clinical proof-of-concept trials to be started. The fit-for-human application prototypes so developed might cut their way further through the formal testing phases until market authorisation and pharmacovigilance are reached (2). The recent success of stabilised and protected mRNA as active pharmaceutical ingredient is a glaring example of nanobiotechnology success. The mRNA in candidate vaccines needs special protection by a nanotechnology delivery system. The 

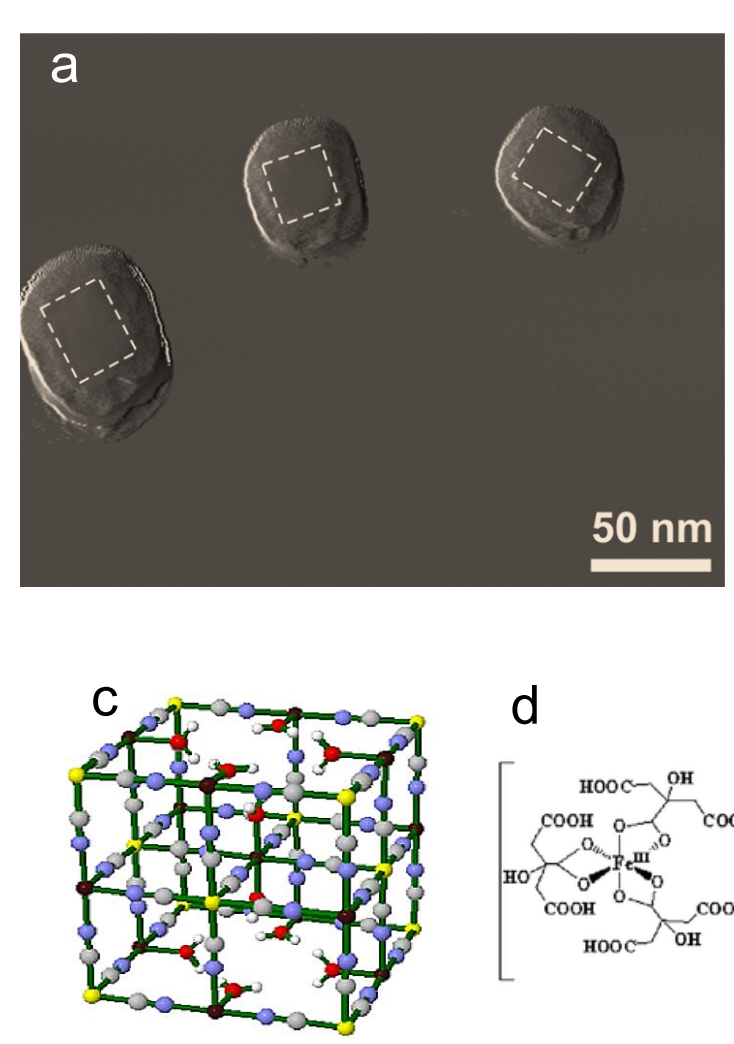

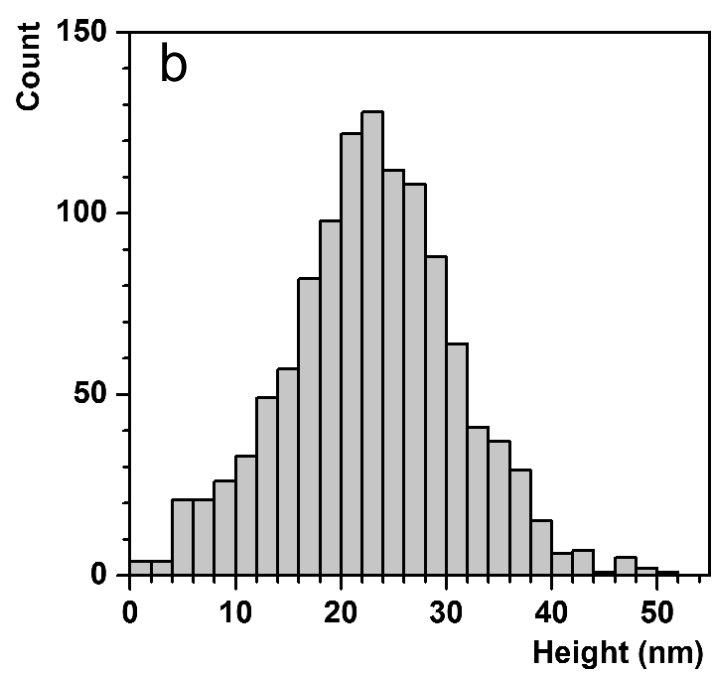

e

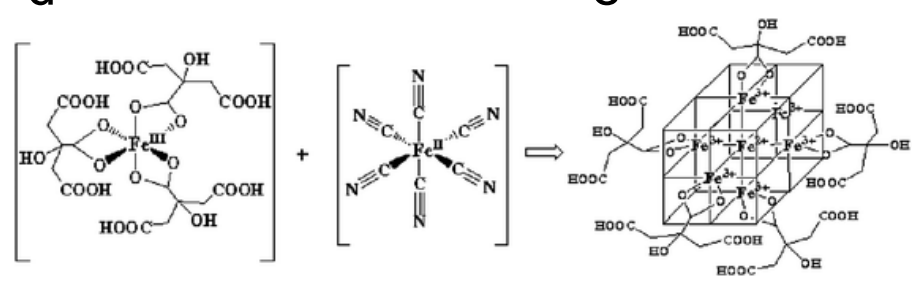

Figure 1. Topographical structure, size distribution and chemical constituents of Prussian Blue nanoparticles (PBNP). a. High-resolution Atomic Force Microscopic (AFM) image of PBNPs. The cuboid shape of individual particles is highlighted by the segmented lines that enclose a flat rectangular area on their surface. b. Distribution of the maximal topographical height obtained by automated particle analysis on 1147 particles. Crystal structure (c), and the schematics (d) and spatial arrangement (e) of coordinated constituting ions of citrate-coated PBNPs. Figure adapted from (12) and (15), copyright the Authors.

so developed and currently administered vaccines are applied world-wide under "emergency use authorisation (EUA)" before a valid marketing authorisation is obtained, even though they have passed Phase III studies. The efficiency and the large application base was largely made available by the use of"lipid nanoparticle" zwitterionic delivery technology that enables the negatively charged RNA to entering the recipient cells and become relased in the acidic lysosomal/endosomal environment to be further translated into protein $(3,4)$. Thus, nanotechnology, and especially nanoparticle technology, has gained new impetus (5). We postulate that new forms of nanoparticle delivery systems will soon be entering clinical phases. By way of examples, such forms are based on different extracellular vesicle technologies $(6,7)$, virus-like particle (VLP) technologies (8), dendrimers, multilayered and multi-component core-shell nanoparticles and the like (4).

Adequate and succcessful translation requires yes/no (or go/ no-go) decisions to be based on quantitative data obtained in iterative cycles at all stages of biotechnology and nanoparticle development $(1,2)$. Iteration involves cycles of measurements and imaging at the macromolecular level at various stages of technological readiness. A finally successful translation thus involves several rounds of back-translation. In vivo wholebody imaging using hybrid molecular imaging methods is now a quintessential tool in drug development. Imaging offers an increase in speed and throughput of candidate molecule pipelines. It feeds back essential information on drug effects and pharmacokinetics first in experimental animal models, and then in humans (2).

Nanoparticle qualities can also be exploited to carry a single platform both for a diagnostic and for a therapeutic role (9, 10). These theranostic platforms usually provide an increment of imaging contrast and therapeutic payloads simultaneously. Their applications are determined by their size, surface charges and surface macromolecular structures (11). During the development of such theranostics, particle size, distributions, degree of polymorphism, surface zeta potential, and drug-/-payload entrapment or release efficiencies have been proven to be the 

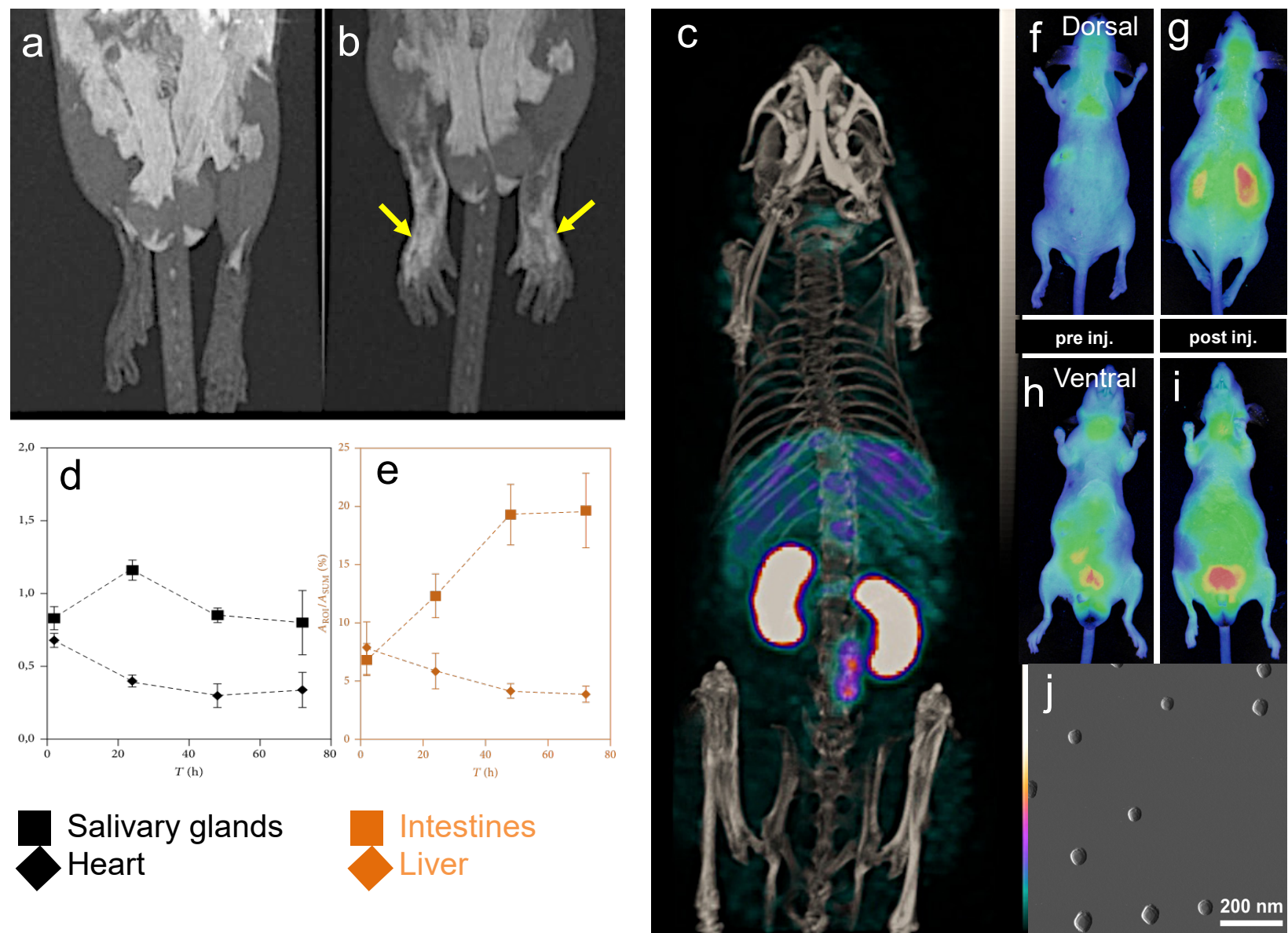

Figure 2. Multimodal contrast-enhancing properties of labeled biocompatible Prussian Blue nanoparticles (PBNP). a. Pre-injection T1-weighted MR image of a mouse injected with PBNP. Subcutaneous injection of $60 \mathrm{uL}$ methylene blue-labeled, polyethyelene-glycol-capped particles in both hindlimbs caused noticeable T1 contrast increase in 24hours after injection. b. Post-injection T1 contrast increase in the hindpaws (yellow arrows). c. A similar PBNP preparation, labeled with 201Tl isotope reaches salivary glands, liver and kidneys in 24 hour post intravenous injection. Maximum intensity projection single photon emission computed tomography/X-ray computed tomography (SPECT/CT) image of the mouse is depicted. CT densities are shown in grayscale, SPECT Activities in the Regions of Interest (ROI) are shown in colour scale in \% proportion of ROI radioactivity to total injected activity. $\mathrm{d}$ and e illustrate the biodistribution of PBNPs over time from SPECT volumes. $\mathrm{f}$ and $\mathrm{h}$ show dorsal and ventral whole-body autofluorescence images, respectively, of a mouse before injection with the PBNP preparation labeled with methylene blue. Fifteen minutes after intravenous injection the fluorescence signal was captured from the entire mouse body, with greates intensities above the kidneys, as shown in post-injection images in $\mathrm{g}$ (dorsal view) and $\mathrm{i}$ (ventral view). $\mathrm{j}$. AFM images of PBNPs injected into animals. Figures adapted and modified from (12) and (15), copyright the Authors.

most important determinants of biodistribution and biomedical effect. Recently, with the advent of extracellular vesicle and VLP biotechnology, the mechanical properties of shells such as elasticity, rigidity and surface functionalisation have also been subjected to investigation as determinants of effect (3).

Reviewing these emerging technologies in a comprehensive manner is beyond the scope of the current treatise; rather, here we present a concept for integrating multi-level, quantitative correlated imaging technologies into the nanobiotechnology discovery and development process. Accordingly, we present development opportunities involving combinations of macromolecular imaging, microscopies and high resolution wholebody in vivo imaging through a sample study $(2,4,12-15)$. The concept, named "3M" for "Molecules-to-Mouse-to-Man", merges correlated bioimaging methodologies aimed at several levels of the biological hierarchical organization.

\section{Nanoparticle characterisation}

The size and shape of individual nanoparticles can be characterized by using atomic force microscopy (AFM). AFM has become the most effective and direct imaging method for measuring the topographical structure and elastic (or viscoelastic) properties of nanoscale macromolecular surfaces and supramolecular assemblies by means of an oscillating cantilever. Automated analysis procedures are available to quantify a large number of parameters associated with the nanoscale objects in the AFM images. We integrate AFM-obtained shape and size measurements into our nanoparticle development pipelines. Such an integration yields faster quality feedback and preparation procedures simpler and quicker than those used in electron 

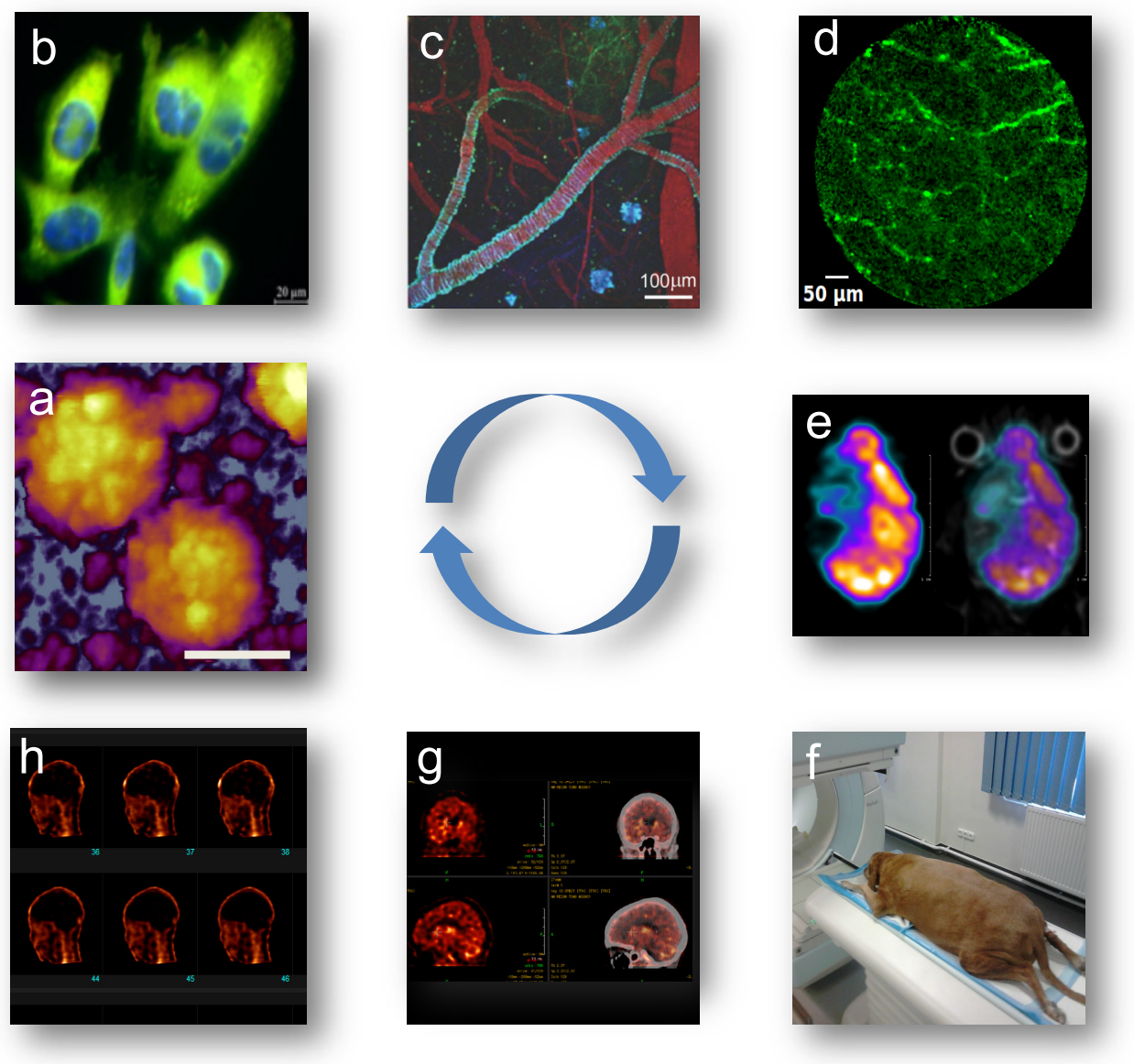

Figure 3. The concept of multi-scale imaging from molecules-to-mose-to-man ("3M"). Logical elements of the 3M pipeline are indicated clockwise, from a through h. a. Height-contrast AFM image of substrate-adsorbed SARS-CoV-2 virions displaying their surface proteins (scale bar, $100 \mathrm{~nm}$ ), b. Fluorescence images of glioma cells loaded with a fluorescent dye (NBD) and Hoechst-blue for nuclear staining, c. In vivo multiphoton fluorescence image of a mouse brain with green fluorescent protein (GFP)-expressing neurons. Texas Red-labeled dextran in red fluorescence was used as a vascular marker, blue fluorescence indicates perivascular amyloid. d. NBD-loaded liposome vascular contrast in a live animal glioma xenograft measured by using confocal fiber optic fluorescence microscopy. e. 99mTc-hydroxymethylydene-paraamino-oxime (HMPAO) perfusion SPECT brain image coupled to gradient echo MRI in a mouse stroke model. f. Experimental setup of a living dog SPECT imaging study. g. HMPAO human brain perfusion SPECT/CT. h. Human bone scan using 99mTc-labeled bisphosphonate.

microscopy. By using AFM both the dehydrated and hydrated nanoparticle shapes can be visualized, therefore their in vivo behavior can be more realistically estimated (16). With AFM it is also possible to monitor changes in the nanoparticle's structure caused by environmental effects "on the fly", for example by temperature using heated stages $(16,17)$. Here we show an example of AFM integration into the testing of Prussian Blue nanoparticles (PBNPs) which we have developed for functional imaging. Besides the standard determination of particle size distribution with dynamic laser light scattering, measurement of zeta potential and collection of electron microscopic images, we carried out an AFM investigation to reveal the topographical structure, size and shape of individual particles. The resulting of PBNP AFM images and measurements are shown in Figure 1.

\section{Measurement of nanoparticle biodistribution}

Prussian Blue nanoparticles (PBNPs) are very attractive for a clinical translational use as radiology and nuclear medicine contrast agents $(12,18)$. The base material Prussian Blue is a clinically approved drug in the treatment of $\mathrm{Cs}$ and $\mathrm{Tl}$ poisoning. All modifications in the PBNP production formulae were made by using clinically compatible, FDA-approved excipients and dyes. PBNPs have been proven to be applicable in a variety of diagnostic pre-clinical modalities, from Magnetic Resonance Imaging (MRI) through Single Photon Emission Computed Tomography (SPECT) or photoacoustic imaging $(12,15,18-20)$. This is largely due to their easy synthesis and their porous structure with a high surface-to-volume ratio (21). PBNPs, capped with polyethylene glycol (PEG), polyvinyl-pyrrolydone (PVP) and/or citric acid are inert and biocompatible. Their payload can be delivered to the targeted tissue by different shell-anchored peptides and proteins, but even their shape plays an important role in reaching the target. In our process of exploration towards a multi-modal theranostic contrast material, we analysed optical imaging, MRI, and ${ }^{201} \mathrm{Tl}$ - isotope radiolabeled PBNPs as a form of chemically stable contrast material with application in SPECT. 
For optical and MR imaging we applied the clinically available fluorescent dye methylene blue $(\mathrm{MB})$ and coated the PBNPs with PVP and PEG. In SPECT studies we determined the quantitative biodistribution of the particles by using ${ }^{201} \mathrm{Tl} \mathrm{ra}-$ diolabeling (Figure 2). All labels were proven to be stable; they remained on the surface of the particles or inside the pores, and thus contributed to an increased image contrast. Prussian Blue nanoparticles developed in our team were shown to display a cuboid shape (Figure 1). We suspect that this shape may have influenced the biodistribution of the PNBNPs as determined by SPECT imaging of the salivary gland uptake. Altogether, we are convinced that the exclusive use of biocompatible and clinically registered materials to construct PBNP nanoparticle families leads to an easier translation to clinical use.

The observation of PBNP properties at the nanoscale (Figure 1) and the organismal scale (Figure 2) lends a strong basis for expanding the concept of across-the-scales imaging towards the molecular level and towards the whole human body. The ultimate aim of this expansion is to reveal as much timeand space-correlated detail as possible about as small a volume element of the living human body as possible. The schematics and logical steps of this "molecules-to-mouse-to-man" (3M) concept are illustrated in Figure 3. The unique insights acquired about a given molecular system or supramolecular assembly (e.g., a virus particle) at the level of the individual particle can be adapted towards understanding their behavior in tissues or experimental animal organisms, so that eventually human diagnostic and therapeutic possibilities can be explored and tested. In the midst of the COVID-19 pandemic, which necessitates the understanding of the pathogenic agent, SARS$\mathrm{CoV}-2$, both at the virion and the tissue and organism levels, the $3 \mathrm{M}$ concept yields special significance.

\section{Conclusions}

Building on a nanoparticle-based theranostic tool development project, here we have proposed the $3 \mathrm{M}$ concept that expands structural and functional imaging across multiplex biological scales from the molecular level, through small-animal organisms, to the entire human body. Starting from information gathered in AFM measurements, we explored the biodistribution of different PBNP formulations. The presented nano- and macroscale imaging measurements applied within a very close timeframe ideally complement each other $(2,12,16,22,23)$. Properties of nanoparticles determining their biodistribution can be tracked and targeted using this combination. High-resolution and high-throughput imaging modalities, such as optical whole body imaging, micro-X-ray-based CT and MRI provide anatomical detail that can be fused with functional image data of the living organism under normal and disease conditions (24). These functional data have been obtained for decades in nuclear medicine using PET and SPECT, which possess unparalleled sensitivity and functionality (2). Combining these methods to understanding microscale and nanoscale properties of living matter enables a more rapid development of diagnostic and therapeutic tools. Furthermore, using back-trans- lation, our zooming-in approach can refine the search results obtained in quantitative in vivo animal multi-modality imaging with high-definition imaging from cells down to organelles and single molecules identified in animal model images. Our approach yields results in early biodistribution or side-effect detection and predictions, as illustrated in nanoparticle development. The molecules-to-mouse-to-man concept will likely provide a unique understanding of correlated high resolution, multi-dimensional information about specific biomolecular processes inside the living human body. We expect that the $3 \mathrm{M}$ concept may greatly enhance biotechnology discoveries and speed up their marketable clinical application.

\section{Acknowledgements}

The research leading to these results has received funding from the European Union`s Horizon 2020 with contract Nr. 739593 (HCEMM). This work was funded by grants from the Hungarian National Research, Development and Innovation Office (K124966 and 135360 to MK; Thematic Excellence Program, TKP-BIOImaging, financed under the 2020-4.1.1-TKP2020 funding scheme). The research was financed by the Higher Education Institutional Excellence Programme of the Ministry for Innovation and Technology in Hungary, within the framework of the Therapeutic Development thematic programme of Semmelweis University.

\section{References}

1. Ansari, M.A., et al., Current Nanoparticle Approaches in Nose to Brain Drug Delivery and Anticancer Therapy - A Review. Current Pharmaceutical Design, 2020. 26(11): p. 1128-1137.

2. Cunha, L., et al., The role of molecular imaging in modern drug development. Drug Discovery Today, 2014. 19(7): p. 936-948.

3. Khurana A, A.P., Khurana I, Allwadhi S, Weiskirchen R, Banothu AK, Chhabra D, Joshi K, Bharani KK, Role of nanotechnology behind the success of mRNA vaccines for COVID-19. Nano Today, 2021.

4. Pascolo, S., Synthetic Messenger RNA-Based Vaccines: From Scorn to Hype. Viruses-Basel, 2021. 13(2).

5. Yetisgin, A.A., et al., Therapeutic Nanoparticles and Their Targeted Delivery Applications. Molecules, 2020. 25(9).

6. Palmai, M., et al., Direct immobilization of manganese chelates on silica nanospheres for MRI applications. Journal of Colloid and Interface Science, 2017. 498: p. 298-305.

7. Varga, Z., et al., Radiolabeling of Extracellular Vesicles with Tc-99m for Quantitative In Vivo Imaging Studies. Cancer Biotherapy and Radiopharmaceuticals, 2016. 31(5): p. 168-173.

8. Chung, Y.H., H. Cai, and N.F. Steinmetz, Viral nanoparticles for drug delivery, imaging, immunotherapy, and theranostic applications. Advanced Drug Delivery Reviews, 2020. 156: p. 214-235.

9. Ghitman, J., et al., Review of hybrid PLGA nanoparticles: 
Future of smart drug delivery and theranostics medicine. Materials \& Design, 2020. 193.

10. Vijayan, V.M., P.N. Vasudevan, and V. Thomas, Polymeric Nanogels for Theranostic Applications: A Mini-Review. Current Nanoscience, 2020. 16(3): p. 392-398.

11. Indoria, S., V. Singh, and M.F. Hsieh, Recent advances in theranostic polymeric nanoparticles for cancer treatment: A review. International Journal of Pharmaceutics, 2020. 582.

12. Forgach, L., et al., Fluorescent, Prussian Blue-Based Biocompatible Nanoparticle System for Multimodal Imaging Contrast. Nanomaterials, 2020. 10(9).

13. Kiss, B., et al., Topography, Spike Dynamics, and Nanomechanics of Individual Native SARS-CoV-2 Virions. Nano Letters, 2021. 21(6): p. 2675-2680.

14. Stephen, Z.R., F.M. Kievit, and M.Q. Zhang, Magnetite nanoparticles for medical MR imaging. Materials Today, 2011. 14(7-8): p. 330-338.

15. Szigeti, K., et al., Thallium Labeled Citrate-Coated Prussian Blue Nanoparticles as Potential Imaging Agent. Contrast Media \& Molecular Imaging, 2018.

16. Baalousha, M. and J.R. Lead, Characterization of natural and manufactured nanoparticles by atomic force microscopy: Effect of analysis mode, environment and sample preparation. Colloids and Surfaces a-Physicochemical and Engineering Aspects, 2013. 419: p. 238-247.

17. Sitterberg, J., et al., Utilising atomic force microscopy for the characterisation of nanoscale drug delivery systems. European Journal of Pharmaceutics and Biopharmaceutics, 2010. 74(1): p. 2-13.F

18. Sharma, P., et al., Multimodal Nanoparticulate Bioimaging Contrast Agents. Cancer Nanotechnology: Methods and Protocols, 2010. 624: p. 67-81.

19. M. Verdaguer, G.S.G., Magnetic Prussian Blue Analogs, in Magnetism: Molecules to Materials, M.D. J. S. Miller, Editor. 2004, M. Wiley-VCH Verlag GmbH \& Co. KGaA. p. 283-346.

20. Shokouhimehr, M., et al., Biocompatible Prussian blue nanoparticles: Preparation, stability, cytotoxicity, and potential use as an MRI contrast agent. Inorganic Chemistry Communications, 2010. 13(1): p. 58-61.

21. Gao, X.R., et al., The Application of Prussian Blue Nanoparticles in Tumor Diagnosis and Treatment. Sensors, 2020. 20(23).

22. Powers, K.W., et al., Research strategies for safety evaluation of nanomaterials. Part VI. Characterization of nanoscale particles for toxicological evaluation. Toxicological Sciences, 2006. 90(2): p. 296-303.

23. Zhang, Y.N., et al., Nanoparticle-liver interactions: Cellular uptake and hepatobiliary elimination. Journal of Controlled Release, 2016. 240: p. 332-348.

24. Tremoleda, J.L., et al., Imaging technologies for preclinical models of bone and joint disorders. Ejnmmi Research, 2011. 1. 